review

\title{
Cancer cachexia-anorexia syndrome and skeletal muscle wasting
}

\author{
Mihaela Jurdana \\ College of Health Care Izola, University of Primorska, Izola, Slovenia
}

\begin{abstract}
Background. Cachexia-anorexia syndrome is a common and important indicator of cancer. It occurs in 30\% to $80 \%$ of cancer patients. Cachexia means "bad condition" and may be present in the early stages of tumor growth, before any signs of malignancy. Cancer cachexia is a syndrome of progressive body wasting, characterized by loss of adipose tissue and skeletal muscle mass. In most cancer patients, cachexia is characterized by anorexia, which implies a failure of food intake, regulated through a complex system of hormones and neuropeptides. A decline in food intake relative to energy expenditure is a fundamental physiologic derangement leading to cancer associated weight loss. The weight loss in patients with cachexia-anorexia syndrome differs from that in caloric starvation or anorexia nervosa. The pathophysiology of cancer cachexia is not fully understood; however, studies have shown that cytokines are important in the alteration of the carbohydrate, lipid and protein metabolism. Cancer, prolonged bed rest, HIV infection and aging are conditions in which muscle wasting is a common feature. An intervention that may potentially attenuate the progression of muscle wasting in cancer patients is resistance exercise training, defined as multiple repetitions of static or dynamic muscular contractions that increase muscle mass.

Conclusions. The main components of the pathological state of cachexia are anorexia and metabolic abnormalities such as fat depletion and muscle protein catabolism. Future developments may concentrate on the molecular abnormalities of cachexia and on examination of the functional benefit of resistance exercise training for cancer related muscle wasting.
\end{abstract}

Key words: cancer cachexia; muscle wasting; cytokines; muscle

\section{Introduction}

Many patients with chronic or end-stage diseases, such as infection, cancer, acquired immunodeficiency syndrome (AIDS), congestive heart failure, cystic fibrosis, tuber-

Received 10 December 2008

Accepted 15 January 2009

Correspondence to: Mihaela Jurdana, PhD, University of Primorska, College of Health Care Izola, Polje 42, 6310 Izola, Slovenia. Phone: +386 566264 69; Fax: +386566264 80; E-mail: mihaela.jurdana@vszi.upr.si culosis, rheumatoid arthritis, and Crohn's disease, develop cachexia. ${ }^{1}$

The word "cachexia" is derived from Greek "kakos" meaning "bad" and "hexis" meaning "condition". Cachexia is characterized by weight loss involving depletion of host adipose tissue and skeletal muscle mass. Weight loss in cancer patients differs from that found in caloric starvation, where body fat is lost preferentially (Table 1). Cachexia is an important cause of mortality in cancer patients, between $10-22 \%$ of all cancer 
Table 1. Characteristics of starvation and cancer cachexia. Cancer associated weight loss arises from the loss of equal amounts of muscle and fat and is characterized by increased catabolism of skeletal muscle and decreased protein synthesis.

\begin{tabular}{ll}
\hline Starvation & Cancer cachexia \\
\hline Mobilization of fat, sparing of skeletal muscle & Equal mobilization of fat and skeletal muscle \\
Decreased basal metabolic rate & Normal or increased basal metabolic rate \\
Liver atrophy & Increased liver size \\
Normal lipoprotein lipase & Reduced lipoprotein lipase \\
Decreased protein breakdown & Increased protein breakadown \\
Reduced glucose turnover & Increased synthesis of acute- phase protein \\
\hline
\end{tabular}

deaths, as well as death from other causes such as infection. ${ }^{2}$ An effective therapy for cachexia should improve the quality of life of cancer patient and it may also be expected to extend the survival time. In general, patients with solid tumors have a higher frequency of cachexia. ${ }^{3}$ About $80 \%$ of patients with upper gastrointestinal cancer and $60 \%$ of patients with lung cancer have had substantial weight loss. ${ }^{3}$ Cachexia is more common in children and elderly patients and becomes more pronounced as the disease progresses.

Tumor growth is associated with profound metabolic and neurochemical alterations. About half of all cancer patients show a syndrome of cachexia, causing the clinical manifestations of anorexia, one of the most common symptoms in advanced cancer. ${ }^{4}$ Anorexia is defined as the loss of desire to eat, which frequently leads to reduced food intake. Based on various diagnostic tools, anorexia has been detected at the point of cancer diagnosis in $13-55 \%$ of patients. ${ }^{5}$ In cancer patients, the development of anorexia is associated with the presence of cachexia, resulting in cancer anorexia-cachexia syndrome. ${ }^{6}$ Symptoms which are usually identified as part of cachexia-anorexia syndrome include weight loss, anorexia, early satiety, muscular weakness and anemia. ${ }^{7}$ Anorexia alone is unlikely to be responsible for the wasting seen in cancer patients, although it may be a contributing factor because the de- gree of weight loss cannot be ascribed completely to reduced food intake. Anorexia and cachexia can co-exist in cancer patients. The muscle wasting observed in cancer patients occurs even in the presence of normal food intake, and increased muscle proteolysis is detectable even before weight loss occurs. ${ }^{8}$ The pathogenesis of anorexia is multifactorial and is related to disturbance of the central physiological mechanisms controlling food intake. The presence of anorexia can be characterized by identification of objective symptoms, including early satiety, taste and smell alterations, meat aversion and nausea/vomiting. ${ }^{9}$

The presence of anorexia is an extremely distressing syndrome, because appetite and the ability to eat have been reported to be the most important factors in the physical and psychological aspects of a patient's quality of life. ${ }^{10}$ In anorexic cancer patients, early satiety together with a reduced appetite has been postulated to be caused by the production of factors by the tumor that exert their effects by acting on the hypothalamic sensory cells. ${ }^{11}$

Loss of appetite can arise from decreased taste and smell of food, as well as from cytokine production. ${ }^{4}$ An alteration in the regulation of feeding, controlled by a complex of hormones and neuropeptides in the ventral hypothalamus, are also involved in cachexia-anorexia. For instance, neuropep- 


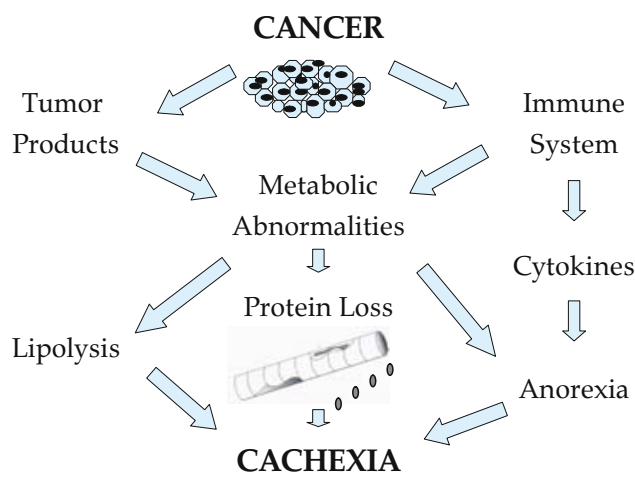

Figure 1. Metabolic abnormalities caused by cancer, resulting in losses of fat and skeletal muscle. Tumors produce factors inducing breakdown of adipose tissue into fatty acids, and protein degradation (amino acids) in skeletal muscle. Cytokines synthesised by tumor or host induced anorexia, the most common symptoms in advanced cancer. Progressive weight loss and skeletal muscle protein loss, profound anorexia characterized Cachexia-Anorexia Syndrome.

tide $\mathrm{Y}$ (NPY) is considered to be among the most potent of the feeding stimulatory peptides. ${ }^{12,13}$ Increased levels of leptin, a hormone secreted by adipocytes, can block NPY and induce satiety. ${ }^{14}$

Cytokines, in particular, appear to play a key role in satiety disturbances, and an emerging view is that anorexia-cachexia syndrome is caused predominantly by cytokines, either produced by cancer cells or released by the immune system of the host as a response to the presence of cancer. ${ }^{14}$

The wasting observed in cancer cachexia can arise from a decreased energy intake and increased energy expenditure (EE - the amount of calories required for a 24-hour period by the body during an active period) or a combination of both. Hypermetabolism and weight loss are significant predictors of decreased survival and they also indicate that weight loss cannot be explained solely by diminished dietary intake. ${ }^{15}$

Patients with cancer have a highly variable change in energy expenditure. Cancer associated weight loss arises from the loss of equal amounts of muscle and fat and is characterized by increased catabolism of skeletal muscle and decreased protein synthesis. ${ }^{16}$ Weight loss can occur during any phase of the disease, and some tumors seem more capable than others of inducing the metabolic changes apparent in cancer cachexia syndrome. In comparison with control groups, patients with malignant disease have been reported to have reduced, normal or elevated energy expenditure. ${ }^{17-19}$ The metabolic abnormalities caused by cancer are shown in Figure 1.

Patients with lung and pancreatic cancer have an increased resting energy expenditure (REE - the amount of calories required for a 24-hour period by the body during a non active period) compared with healthy control subjects. ${ }^{20,21}$ However, patients with gastric and colorectal cancer were reported to have no elevation of REE. ${ }^{20}$ Cachexia can occur even with a normal EE, which suggests that tumor and host factors play an important role in depletion of body lipids (FAT) and protein during the process of cachexia.

The metabolic profile of cachexia is not the same as that of caloric starvation. ${ }^{7}$ During the first few days of starvation, glucose utilization by the brain and erythrocytes necessitates depletion of liver and muscle glycogen and increased glucose production by the liver, using gluconeogenic amino acids derived from catabolism of muscle. This phase is replaced in longterm starvation by the use of fat as a fuel, in which free fatty acids released from adipose tissue are converted into ketone bodies, which are utilized for energy by peripheral tissues and, eventually, to some extent by the brain. This leads to prolonged conservation of muscle mass. ${ }^{6}$ In contrast, in cancer cachexia, there is equal loss of both fat and muscle, so that for a given degree of weight loss there is more loss of muscle in a patient with cancer cachexia than in caloric starvation. ${ }^{22}$ In starving persons, the body 
quickly adapts to the lack of nutrients by decreasing its EE and by oxidizing store lipids for energy. This metabolic adaptation to a decreased food intake is critical for maintaining a functional muscle mass. Cancerbearing hosts, however, fail to develop such metabolic adaptation and thus continue to deplete their skeletal muscle proteins. ${ }^{6}$ Metabolic abnormalities in cancer cachexia are described in Figure 1.

\section{The role of cytokines}

Cytokines are polypeptides synthesised and released primarily by activated monocytes and macrophages. They are also produced by some types of tumor cells. ${ }^{23}$

Skeletal muscle plays an important role in the immune system as one of the producers of cytokines. Cytokines are primarily responsible for cell-to-cell communication and stimulate the arrival of lymphocytes, neutrophils, monocytes and other healer cells to the injury site to repair the injured tissue. ${ }^{24}$ Three important cytokines, tumor necrosis factor- $\alpha$ (TNF- $\alpha$ ), interleukin 1 (IL-1) and interleukin 6 (IL-6), are responsible for protein breakdown in skeletal muscle and increased production of prostaglandins. Cytokines are also involved in muscle hypertrophy and in the muscle regeneration process. ${ }^{25}$

Numerous cytokines, including (TNF- $\alpha)$, (IL-1), (IL-6), interferon $\gamma($ IFN- $\gamma$ ) and leukemia-inhibitory factor (LIF), play an important role in the etiology of cancer cachexia. Such cytokines may be produced by tumor or host tissue and are characterized by the induction of anorexia and a decrease in the clearing enzyme lipoprotein lipase. ${ }^{6}$

Cytokines are implicated in changes in sensory function in the chorda tympani (involved in the transduction of taste), leading to alterations in taste and in specific food preferences. ${ }^{4}$ Patients with end stage cancer have altered taste thresholds with respect to the bitter modality and these changes are most apparent in patients with higher concentrations of C-reactive protein, IL $\beta-1$, IL-6 and TNF- $\alpha$. The odor threshold was also lower in these patients than in healthy subjects. ${ }^{26}$ The described cytokines also sensitize the vagal afferent nerve fibers, resulting in increased activation of the mechanisms that mediate sensations of fullness and thus contribute to the process of satiety. ${ }^{26}$ Cytokines may inhibit feeding by causing nausea and vomiting and also by decreasing gastric motility and gastric emptying, intestinal motility or by modifying gastric acid secretion. Their effects may result from direct action on the gastrointestinal system or indirect effects mediated by cytokines (IL $\beta-1$, IL-2, IFN- $\gamma$ and TNF- $\alpha$ ) on the central nervous system. ${ }^{12,27-33}$ Cytokines can also interact with prostaglandins (members of a group of lipid compounds that are derived enzymatically from fatty acids and have important functions in the body) and corticotrophin-releasing hormone (CRH), a polypeptide hormone and neurotransmitter involved in the stress response to inhibit gastric emptying. ${ }^{29,30}$

Cytokine interaction with neuropeptides may induce anorexia and can also modify serotonin and catecholamine pathways in the central and peripheral nervous system. Cytokines; TNF- $\alpha$, IL-1, IL- 6 and IFN- $\gamma$ have been implicated in the induction of cancer related muscle wasting. ${ }^{34}$

\section{Interleukin-1 (IL-1)}

IL-1 was observed to induce anorexia, weight loss and hypoalbuminemia in mice ${ }^{35}$ and satiety in rats as a result of activation of gluco-sensitive neurons in the ventromedial nucleus of the hypothalamus. ${ }^{36,37}$ Otterness et al. ${ }^{38}$ and Mrosovsky et al. ${ }^{39}$ observed cachexia-causing effects of IL1 when it is administrated to animals. Transfection of a cachectic tumor cell line 
(colon-26) with the gene for IL-1 receptor antagonist failed to abolish the capacity of the tumor to produce cachexia. ${ }^{40}$ These results confirmed a role of this cytokine in the induction of tissue wasting in cancer.

\section{Tumor necrosis factor alpha (TNF- $\alpha$ )}

Many of the metabolic disturbances associated with IL-1 are similar to those of TNF- $\alpha$, a possible cachectic factor. Administration of TNF- $\alpha$ to laboratory animals induces a state of cachexia, with anorexia and depletion of adipose tissue and lean body mass. Its effects are mediated centrally and in the gastrointestinal tract. TNF- $\alpha$ infusions directly reduce gastric emptying and peristalsis, ${ }^{41}$ they can induce lipolysis and inhibit lipid synthesis, ${ }^{42-46}$ and increase proteolysis in peripheral muscle. ${ }^{47}$ TNF- $\alpha$ has been shown in several studies to activate muscle protein degradation directly and induces IL6 release. ${ }^{48}$ It has been demonstrated that IL- 6 can impair TNF- $\alpha$ expression in cardiac muscle; one potential role of IL- 6 expression in contracting skeletal muscle is therefore to downregulate TNF- $\alpha$ expression. ${ }^{49}$

Llovera et al. ${ }^{50}$ demonstrated that TNF- $\alpha$ administration to healthy, cancer free rats brought about an enhanced rate of degradation of skeletal muscle protein, even though body weight loss was not apparent in the animals.

Hyperexpression of TNF- $\alpha$ has been identified as one of the key cytokine responses involved in cachexia. ${ }^{51} \mathrm{TNF}-\alpha$ appears to influence several other abnormalities present during cancer cachexia: adipose and muscle wasting, insulin resistance, increased thermogenesis, and alteration in lipid and protein metabolism. ${ }^{52,53}$

\section{Interleukine - 6 (IL-6)}

The role of IL-6 in the development of cancer cachexia has mainly been provided from animal studies involving the use of murine colon-26 adenocarcinoma model. Evidence of a causative role of IL-6 in the pathogenesis of anorexia and cachexia comes from experiments reporting that treatment with anti-mouse IL-6 antibody was successful in reversing the key parameters of anorexia in mice bearing adenocarcinoma. ${ }^{54,55}$

Elevated serum concentration of IL6 has been reported in cancer patients. IL-6 increased in lung cancer patients and has been identified as a mediator of cachexia by the growth of a uterine cervical carcinoma (Yomoto) in nude mice. ${ }^{56}$ An elevated level of serum IL-6 has been reported in patients with colon cancer and in acute-phase response, ${ }^{57}$ however, since all patients had lost weight, it is difficult to associate this elevation with the induction of cachexia.

Muscle atrophy has been observed in IL-6 transgenic mice, and another study reported that administration of IL-6 to rats acutely activated both total and myofibrillar protein degradation in skeletal muscle. ${ }^{58} \mathrm{It}$ has been demonstrated that contracting human skeletal muscle released IL- 6 but not TNF- $\alpha$, because it negatively affects glucose uptake in skeletal muscle. ${ }^{59}$

Glucocorticoids can prevent the stimulatory effects of proinflammatory factors on IL-6 secretion, which in general stimulate proliferation at the earliest, myoblast stage of muscle formation. Prelovsek et al. ${ }^{48}$ reported that a high dexametasone concentration prevents the stimulatory effects of TNF- $\alpha$ and LPS on IL- 6 secretion from the precursors of human muscle regeneration. It results in prevention of myoblast proliferation, leading to a reduced final mass of the regenerated muscle.

The results of human and animal studies strongly implicate IL-6 in the cachectic process. IL-6 probably does not act alone but may either induce or act in synergy with other cachectic factors. 


\section{Interferon gamma (IFN- $\gamma)$}

In mice bearing Lewis lung tumors, the development of tumors is associated with IFN- $\gamma$ production and with progressive weight loss. IFN- $\gamma$ antibodies counteract the wasting syndrome observed in cancer cachexia. ${ }^{60}$ In rats that had received transplants of MCG 101 sarcoma, anti-IFN- $\gamma$ antibody reduced weight loss and anorexia but the treatment was partial and short-lived, suggesting that IFN- $\gamma$ may not be the sole mediator. ${ }^{61}$ Such a result should not be interpreted to mean that IFN- $\gamma$ by itself can induce cachexia, since both IFN- $\gamma$ release and the presence of tumor cells were found to be required.

\section{Leukemia- inhibitory factor (LIF)}

LIF is proposed to be a mediator of cachexia through its ability to decrease lipoprotein lipase activity. LIF plays an important role in the development of cancer cachexia syndrome observed in melanoma-bearing nude mice. The expression of LIF mRNA was examined in four melanoma xenografts, SEKI, G361, A375 and MEWO, in nude mice. SEKI- and G361-bearing nude mice developed cancer cachexia syndrome, and their body weights decreased. A375- and MEWO-bearing nude mice, however, did not develop the syndrome. Northern blot analysis revealed that G361 as well as SEKI expressed a large amount of LIF mRNA, but A375 and MEWO did not, suggesting a close relationship between the expression of LIF mRNA and the development of the syndrome. ${ }^{62}$

\section{Proteolysis-inducing factor (PIF)}

Proteolysis-Inducing Factor, which induces muscle wasting, was purified from cachexia-inducing MAC-16, a murine adenocarcinoma. The exogenous administration of PIF to healthy mice resulted in a $50 \%$ decrease in muscle protein synthesis and a $50 \%$ increase in muscle protein degradation. ${ }^{63}$

A PIF of identical characteristics and molecular weight was detected in the urine of persons losing weight due to pancreatic or gastrointestinal cancers but not in the urine of weight-stable patients with cancer or weight-losing non-cancer patients. ${ }^{64}$

PIF appears to induce muscle wasting via activation of the ubiquitin proteosome pathway. ${ }^{65}$ It seems unlikely that any of the cytokines alone are able to explain the complex mechanism of wasting seen in cancer cachexia, and other factors must be involved.

\section{Effects of cachexia on skeletal muscle}

Cancer cachexia is associated with perturbations in protein metabolism, leading to significant wasting of tissue proteins. Muscle wasting results from an imbalance between the rates of muscle protein synthesis and degradation. Body composition analysis shows that skeletal muscle is the major site of protein loss in patients with solid (non hematological) tumors. ${ }^{66}$ There are also changes in the concentration of plasma amino acids, and most studies report a decrease in gluconeogenic amino acids, in contrast with severe malnutrition, in which the concentration of branched-chain amino acids in plasma is normal or even increased. Protein degradation of amino acid results in the release of amino acids, particularly alanine and glutamine. The former is channeled to the liver for gluconeogenesis and APP (acute phase protein) synthesis, whereas glutamine is taken up by the tumor to sustain energy and nitrogen demands. ${ }^{1}$

Intracellular protein breakdown can be mediated by three pathways; lysosomal, $\mathrm{Ca}^{2+}$-dependent and ATP-ubiquitin dependent proteolytic pathways. All three 
pathways may be involved in cachexia, although the ubiquitin-dependent system is considered to be the most important and has been most studied. ${ }^{67}$ Recent evidence suggests that PIF and TNF- $\alpha$, but not other cytokines, can induce expression of the key regulatory components of this pathway. ${ }^{65,68}$

TNF- $\alpha$ induces muscle wasting via inhibition of pathways involved in muscle cell differentiation and regeneration. ${ }^{69}$ Exposure of myocites to TNF- $\alpha$ activates the transcription factor-nuclear factor kappa B

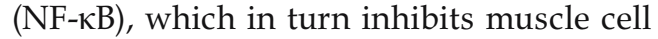
differentiation by suppressing the synthesis of MyoD, a transcriptional factor that is essential for muscle cell differentiation and for the repair of damaged muscle tissue. ${ }^{69}$ The activation of NF- $\mathrm{KB}$ is also involved in upregulation of cytokine synthesis, which can contribute to paracrine effects of cytokines on skeletal muscle tissue, as previously described. Cytokine-induced skeletal muscle wasting is probably a multifactorial process, involving increased protein degradation and reduced myocite regeneration and repair. ${ }^{70}$

The consequence of skeletal muscle wasting is fatigue. Atrophy of skeletal muscle leads to asthenia and muscle weakness, which causes a reduction of physical activity level in affected persons. This leads to more muscle deconditioning and atrophy, which in turn aggravates the feeling of fatigue.

Physical exercise training may potentially attenuate muscle wasting and/or reduce fatigue in cancer patients. Data obtained from healthy humans and from experimental animals demonstrate that regular endurance exercise training of submaximal intensity (below maximal heartbeat) increases muscle endurance and resistance to fatigue. ${ }^{71}$ Resistance exercise training also increases the mass of healthy muscles and attenuates muscle wasting associated with some catabolic conditions. Resistance ex- ercise training in cancer patients increases muscle mass by accelerating the rate of protein synthesis and by attenuating muscle protein breakdown and/or muscle wasting. The effect of resistance exercise training on increased muscle mass in cancer has not been adequately studied.

A mouse model of cancer cachexia (mice bearing the colon-26 adenocarcinoma) was used to test the hypothesis that resistance training, performed by electrical stimulation of the motor nerve would attenuate cancer-related wasting in the contracting muscles. ${ }^{72}$ The results of the study demonstrate that resistance training attenuates wasting of the extensor digitorum longus muscle (EDL) in tumor-bearing mice. This attenuation of wasting was paralleled by an increase in muscle weight, which was due to an increase in the actual mass of muscle and not merely due to edema. The findings of this study also suggested that the dose of training that attenuates wasting of the EDL muscle in tumor-bearing mice is not sufficient to induce hypertrophy in the EDL muscle in the control non-tumor-bearing mice. These data show that wasted muscles respond differently than non-wasted muscles to exercise training. Attenuation of muscle wasting may be easier to achieve than the induction of hypertrophy of healthy nonwasted muscles, which suggests that persons with wasted muscle may not need to exercise as vigorously in order to attenuate wasting of their muscles.

The loss of skeletal muscle mass that accompanies cancer cachexia is associated with changes in muscle functional properties, with a shift in muscle fiber type distribution or a shift in myosin isoform expression. Myosin heavy chain (MHC) and myosin light chain (MLC) expression are characterized by a decrease in the phenotypic expression of "slow" myosine isoforms (type I MHC and slow forms of MLC) and an increase in the phenotypic 
expression of "fast" myosine isoforms (type II MHC and fast MLC isoforms) in the soleus muscle. ${ }^{68}$ These changes are likely to have a significant impact on the functional properties of muscles during cachexia. The exact mechanism of this effect on myosin isoform expression is unknown at present. Evidence suggests that the problem is a multifactorial process mediated by host-released and tumor-released factors. The activation of pro-inflammatory cytokines and various proteolytic pathways, particularly the ubiquitin proteasome pathway within skeletal muscle may be responsible for the problem. ${ }^{69}$ These factors apparently disturb the balance between the rates of skeletal muscle protein synthesis and muscle protein degradation, leading to muscle protein depletion and muscle wasting. Wasting of skeletal muscle contributes to muscle weakness, fatigue and to the morbidity and mortality of cancer.

\section{Conclusions}

There is a high incidence of cancer cachexia-anorexia syndrome in cancer patients. This syndrome is observed in $80 \%$ of patients in advanced stage cancer. Cachexia is characterized by wasting of skeletal muscle and depletion of host adipose tissue, while anorexia is associated with persistent loss of appetite and decrease in food intake. Both induce weight loss that differs from caloric starvation.

The cachectic state is particularly problematic in cancer. It typifies a poor prognosis and often lowers responses to chemotherapy and radiation treatment. ${ }^{74}$ Radiation damages mitotically active muscle satellite cells, prevents compensatory hypertrophy of skeletal muscle, and prevents small fiber formation. ${ }^{75}$

Skeletal muscle wasting during cancer cachexia is well documented, and resist- ance exercises should be examined as a potential intervention for attenuating cancerinduced muscle wasting. Exercise-induced changes of skeletal muscle mass should be pursued in terms of changes in muscle protein synthesis and muscle protein degradation pathways in the exercised versus the unexercised muscles, both of which have been implicated in cancer induced muscle wasting.

Exercise that induces attenuation of muscle wasting is a result of an increase in muscle protein synthesis or a decrease in muscle protein breakdown. Its mechanisms need to be explored in future research. It would be important to examine the functional benefit of training-induced attenuation of cancer-related muscle wasting.

The intervention of preserving muscle mass has an important clinical implication, since it improves the prognosis and the quality of life of cachetic cancer patients.

\section{References}

1. Tisdale MJ. Pathogenesis of Cancer Cachexia. J Support Oncol 2003; 1: 159-68.

2. Inagaki J, Rodriguez V, Bodey GP. Proceedings: causes of death in cancer patients. Cancer 1974; 33: 568-73.

3. DeWys WD. Anorexia as a general effect of cancer. Cancer 1972; 45: 2013-19.

4. Perboni S, Inui A. Anorexia in cancer: role of feedeing-regulatory peptides. Phil Trans R Soc B 2006; 361: 1281-89.

5. Geels P, Eisenhauer E, Bezjak A, Zee B, Day A. Palliative effect of chemotherapy: objective tumor response is associated with symptom improvement in patients with metas tatic breast cancer. J Clin Oncol 2000; 18: 2395-405.

6. Tisdale MJ. Biology of cachexia. J Natl Cancer Inst 1997; 89: 1763-73.

7. Dahele M, Fearon KC. Research methodology: cancer cachexia syndrome. Palliative Medicine 2004; 18: 409-17. 
8. Bossola M, Muscaritoli M, Costelli P, Bellantone R, Pacelli F, Busquets S, et al. Increased muscle ubiquitin mRNA levels in gastric cancer patients. Am J Physiol Regul Integr Comp Physiol 2001; 280: R1 518-23.

9. Rossi Fanelli F, Cangiano C, Ceci F, Cellerino R, Franchi F, Menichetti ET, et al. Plasma tryptophan and anorexia in human cancer. Eur J Cancer Clin Oncol 1986; 22: 89-95.

10. Padilla GV. Psychological aspects of nutrition and cancer. Surg Clin North Am 1986; 66: 1121-35.

11. Knoll J. Endogenous anorectic agents-satietins. Annu Rev Pharmacol Toxicol 1988; 28: 247-68.

12. Plata-Salaman CR. Immunoregulators in the nervous system. Neurosci Biobehav 1991; 5: 185-215.

13. Inui A. Cancer anorexia-cachexia syndrome: are neuropeptides the key? Cancer Res 1999; 59: 4493501.

14. Inui A. Cancer Anorexia-Cachexia Syndrome: current issues in research and management. CA Cancer J Clin 2002; 52: 72-91.

15. Bosaeus I, Daneryd P, Lundholm K. Dietary intake, resting energy expenditure, weight loss and survival in cancer patients. J Nutr 2002; 132 (suppl): S 3465-S 3466.

16. Cohn SH, Gartenhaus W, Sawitsky A, Zanzi I, Vaswani A, Ellis KJ. Compartmental body composition of cancer patients by measurement of total body nitrogen, potassium, and water. Metabolism 1981; 30: 222-9.

17. Knox CS, Crosby CO, Fuerer ID, Buzby GP, Cliffor MD, Mullen JL. Energy expenditure in malnourished cancer patients. Ann Surg 1983; 197: 152-62.

18. Nixon DW, Kutner M, Heymsfield S, Foltz AT, Carty C, Seitz S, et al. Resting energy expenditure in lung and colon cancer. Metabolism 1988; 37: 1059-64.

19. Fredrix, EW, Soeters PB, Wouters EF, Deerenberg IM, Von-Meyerfeldt MF, Saris WH. Effect of different tumor types on resting energy expenditure. Cancer Res 1991; 51: 6138-41.

20. Fredix EW, Saris WH, Soeters PB, Wouters EF, Kester AD, von Meyenfeldt MF, et al. Estimation of body composition by bioelectrical impedance in cancer patients. Eur J Clin Nutr 1990; 44: 749-52.

21. Falconer JS, Fearon KC, Plester CE, Ross JA, Carter DC. Cytokines, the acute-phase response, and resting energy expenditure in cachectic patients with pancreatic cancer. Ann Surg 1994; 219: 325- 31.
22. Moley JF, Aamodt R, Rumble W, Kaye W, Norton JA. Body cell mass in cancer bearing and anorexia patients. J Parenter Enteral Nutr 1987; 11: 219-22.

23. Ohnuma T. Anorexia and cachexia. In: Kufe, Morton, Weichselbaum, editors. Cancer Medicine. Baltimore, MD: Williams \& Wilkins; 1997. p. 3091110.

24. Pedersen BK. Exercise immunology. New York: Chapman \& Hall; 1997.

25. Adams GR, Haddad F. The relationship among IGF-1, DNA content, and protein accumulation during skeletal muscle hypertrophy. J Appl Physiol 1996; 81: 2509-16.

26. Richardson RA, Davidson HI. Nutritional demands in acute and chronic illness. Proc Nutr Soc 2003; 62: 777-81.

27. Van Miert AS, Kaya F, Van Duin CT. Changes in food intake and forestomach motility of dwarf goats by recombinant bovine cytokines IL-1beta, IL-2 and IFN-gamma. Physiol Behav 1992; 52: 859-64.

28. Montuschi P, Tringali G, Curro D, Ciabattoni G, Parente L, Preziosi P, et al. Evidence that IL-1beta and tumor necrosis factor inhibit gastric fundus motility via the 5-lipoxygense pathway. Eur J Pharmacol 1994; 252: 253-60.

29. Suto G, Kiraly A, Plourde V, Tache Y. Intravenous IL-1beta induced inhibition of gastric emptying: involvement of central corticotrophin-releasing factor and prostaglandin pathways in rats. Digest 1996; 57: 135-40.

30. Suto G, Kiraly A, Tache Y. IL-1b inhibits gastric emptying in rats: mediation through prostaglandin and corticotropin-releasing factor. Gastroenterol 1994; 106: 1568-75

31. Sonti G, Ilyin SE, Plata-Salaman CR. Neuropeptide $\mathrm{Y}$ blocks and reverses IL-1b induced anorexia in rats. Peptides 1996; 17: 517-20.

32. Chrousos GP, Torpy DJ, Gold PW. Interactions between the hypothalamic- pituitary-adrenal axis and the female reproductive system: clinical implications. Ann Intern Med 1998; 129: 229-40.

33. Shimon I, Yan X, Ray DW, Melmed S. Cytokine dependent gp 130 receptor subunit regulates human fetal pituitary adrenocorticotropin hormone and growth hormone secretion. J Clin Invest 1997; 100: 357-63.

34. Argiles JM, Costelli P, Carbo N, Pallares-Trujillo J, Lopez-Soriano FJ. Tumor growth and nitrogen metabolism in the host. Int J Oncol 1999; 14: 479-86. 
35. Moldawer LL, Andersson C, Gelin J, Lundholm KG. Regulation of food intake and hepatic protein synthesis by recombinant-derived cytokines. Am J Physiol 1988; 254: G 450-6.

36. Laviano A, Meguid MM, Yang ZJ, Gleason JR, Cangiano C, Rossi Fanelli F. Cracking the riddle of cancer anorexia. Nutrit 1996; 12: 706-10.

37. Laviano A, Gleason JR, Meguid MM, Yang ZJ, Cangiano C, Rossi Fanelli F. Effects of intra-VMN mianserin and IL-1ra on meal number in anorectic tumor-bearing rats. J Investig Med 2000; 48: 40-8.

38. Otterness IG, Seymour PA, Golden HW, Reynolds JA, Daumy GO. The effects of continuous administration of murine interleukin-1alpha in the rat. Physiol Behav 1988; 43: 797-804.

39. Mrosovsky N, Molony LA, Conn CA, Kluger MJ. Anorexic effects of interleukin in the rat. Am J Physiol 1989; 257: R 1315-21.

40. Yasumoto K, Mukaida N, Harada A, Kuno K, Akiyama M, Nakashima E. Molecular analysis of the cytokine network involved in cachexia in colon 26 adenocarcinoma -bearing mice. Cancer Res 1995; 55: 921-7.

41. Bodnar RJ, Pasternak GW, Sha P, Stock MJ. Medication of anorexia by human recombinant tumor necrosis factor through a peripheral action in the rat. Cancer Res 1989; 49: 6280-84.

42. Kawakami M, Cerami A. Studies of endotoxin-induced decrease in lipoprotein lipase activity. J Exp Med 1981; 154: 631-7.

43. Feingold KR, Grunfeld C. Tumor necrosis factoralpha stimulates hepatic lipogenesis in the rat in vitro. J Clin Invest 1987; 80: 184-90.

44. Pape ME, Kim KH. Effect of tumor necrosis factor on acetyl-coenzyme A carboxylase gene expression and pre-adipocyte differentiation. $\mathrm{Mol}$ Endocrinol 1988; 2: 395-403.

45. Semb H, Peterson J, Tavernier J, Olivecrona T. Multiple effects of tumor necrosis factor on lipoprotein lipase in vivo. J Biol Chem 1987; 262: 8390-4.

46. Strassmann G, Fong M, Kenny JS, Jacob CO. Evidence for the involvement of interleukin 6 in experimental cancer cachexia. J Clin Invest 1992; 89: 1681-4.

47. Argiles JM, Garcia-Martinez C, Liovera M, LopezSoriano FJ. The role of cytokines in muscle wasting: its relation with cancer cachexia. Med Res Rev 1992; 12: 637-52.
48. Prelovsek O, Mars T, Jevsek M, Podbregar M, Grubic Z. High dexamethasone concentration prevents stimulatory effects of TNF-alpha and LPS on IL-6 secretion from the precursors of human muscle regeneration. Am J Physiol Regul Integr Comp Physiol. 2006; 291(6): 1651-6.

49. Steensberg A, Keller C, Starkie RL, Osada T, Febbraio MA, Pedersen BK. IL-6 and TNF-alpha expression in, and release from, contracting human skeletal muscle. Am J Physiol Endocrinol Metab 2002; 283(6): 1272-8.

50. Llovera M, Lopez-Soriano FJ, Argiles JM. Effects of tumor necrosis factor- on muscle protein turnover in female Wistars rats. J Natl Cancer Inst 1993; 85: 1334-9.

51. Darling G, Fraker DL, Jensen JC, Gorschboth CM, Norton JA. Cachectic effects of recombinant human tumor necrosis factor in rats. Cancer Res 1990; 50: $4008-13$.

52. Argiles JM, Alvarez B, Lopez - Sorriano FJ. The metabolic basis of cancer cachexia. Med Res Rev 1997; 17: 477-98.

53. Bianchi A, Bruce J, Cooper AL, Childs C, Kohli M, Morris ID, et al. Increased brown adipose tissue activity in children with malignant disease. Horm Metab Res 1989; 21: 640-1.

54. Strassmann G, Jacob CO, Evans R. Mechanisms of experimental cancer cachexia. Interaction between mononuclear phagocytes and colon-26 carcinoma and its relevance to IL-6 mediated cancer cachexia. J Immunol 1992; 148: 3674-8.

55. Tsujinaka T, Fujita J, Ebisui C. Interleukin 6 receptor antibody inhibits muscle atrophy and modulates proteolytic systems in interleukin 6 transgenic mice. J Clin Invest 1996; 97: 244-9.

56. Tamura S, Fujimoto-Ouchi K, Mori K, Endo M, Matsumoto $\mathrm{T}$, Eden $\mathrm{H}$, et al. Involvement of human interleukin 6 in experimental cachexia induced by a human uterine carcinoma xenograft. Clin Cancer Res 1995; 1: 1353-8.

57. Fearon KC, McMillan DC, Preston T, Winstanley P, Cruickshank AM, Shenkin A. Elevated circulating interleukin-6 is associated with an acute phase response but reduced fixed hepatic protein synthesis in patients with cancer. Ann Surg 1991; 213: 26-31.

58. Goodman MN. Interleukin-6 induces skeletal muscle protein breakdown in rats. Proc Soc Exp Biol Med 1994; 205: 182-5. 
59. Pedersen BK, Febbraio MA. Muscle as an Endocrine Organ: Focus on Muscle-Derived Interleukin-6. Physiol Rev 2007; 88: 1379-1406.

60. Matthys P, Heremans H, Opdenakker G, Billiau A. Anti-interferon-gamma antibody treatment growth of Lewis lung tumors in mice and tumorassociated cachexia. Eur J Cancer. 1991; 27: 182-7.

61. Langstain HN, Doherty GM, Fraker DL, Buresh C, Norton JA. The roles of -interferon and tumor necrosis factor in an experimental rat model of cancer cachexia. Cancer Res 1991; 51: 2302-6.

62. Mori M, Yamaguchi K, Honda S, Nagasaki K, Ueda M, Abe O, et al. Cancer Cachexia Syndrome Developed in Nude Mice Bearing Melanoma Cells Producing Leukemia-inhibitory Factor. Cancer Res 1991; 51: 6656-9.

63. Lorite MJ, Cariuk P, Tisdale MJ. Induction of muscle protein degradation by a tumor factor. $\mathrm{Br} J$ Cancer 1997; 76: 1035-40.

64. Todorov PT, McDevitt TM, Cariuk P, Coles B, Deacon M, Tisdale MJ. Induction of muscle protein degradation and weight loss by a tumor product. Cancer Res 1996; 56: 1256-61.

65. Hussey HJ, Todorov PT, Field WN, Inagaki N, TanakaY, Ishitsuka $\mathrm{H}$, et al. Effect of a fluorinated pyrimidine on cachexia and tumor growth in murine cachexia models: relationship with a proteolysis inducing factor. Br J Cancer 2000; 83: 56-62.

66. Cohn SH, Gartenhaus W, Sawitsky A, Rai K, Zani I, Vaswani A, et al. Compartmental body composition of cancer patients by measurement of total body nitrogen, potassium, andwater. Metabolism 1981; 30: 222-9.

67. Argiles JM, Moore-Carrasco R, Fuster G, Busquets S, Lopez Soriano FJ. Cancer cachexia: the molecular mechanisms. Int J Biochem Cell Biol 2003; 35: 405-9.

68. Mitch WE, Goldberg AL. Mechanisms of skeletal muscle wasting: The role of the ubiquitin-proteasome pathway. N Engl J Med 1996; 335: 1897-1905.

69. Guttridge DC, Mayo MW, Madrid LV, Wang C, Baldwin AS. NF- B-induced loss of MyoD messenger RNA: possible role in muscle decay and cachexia. Science 2000; 298: 2363-7.

70. Tisdale MJ. Biomedicine: protein loss in cancer cachexia. Science 2000; 289: 2293-4.

71. Spina RJ, Chi MMY, Hopkins MG, Nemeth PM, Lowry OH, Holloszy JO. Mitochondrial enzymes increase in muscle in response to 7-10 days of cycle exercise. J Appl Physiol 1996; 80: 2250-4.
72. Al-Majid S, McCarthy DO. Cancer-induced fatigue and skeletal muscle wasting: the role of exercise. Biol Res Nurs 2001; 2: 186-97.

73. Diffee GM, Kalfas K, Al-Majid S, McCarthy DO. Altered expression of skeletal muscle myosin isoforms in cancer cachexia. Am J Physiol Cell Physiol 2002; 283: C 1376-82.

74. Tisdale MJ. Cachexia in cancer patients. Nat Rev Cancer 2002; 2: 862-71.

75. Jurdana M. Radiation effects on skeletal muscle. Radiol Oncol 2008; 42: 15-22. 